Citation: A. Villarrubia-Martínez, I. Aguaded-Gómez, Á. Delgado-Ponce (2021) Contenidos infantiles convergentes, identidad latinoamericana y los desafíos de las televisiones públicas. Media Education 12(1): 125-133. doi: $10.36253 / \mathrm{me}-10270$

Received: December, 2020

Accepted: May, 2021

Published: May, 2021

Copyright:@2021A. Villarrubia-Martínez, I. Aguaded-Gómez, Á. Delgado-Ponce. This is an open access, peer-reviewed article published by Firenze University Press (http://www.fupress.com/me) and distributed under the terms of the Creative Commons Attribution License, which permits unrestricted use, distribution, and reproduction in any medium, provided the original author and source are credited.

Data Availability Statement: All relevant data are within the paper and its Supporting Information files.

Competing Interests: The Author(s) declare(s) no conflict of interest.

\section{Contenidos infantiles convergentes, identidad latinoamericana y los desafíos de las televisiones públicas}

\author{
Convergent contents for children's television, Latin American \\ identity, and the challenges of public service television
}

\author{
Andrea Villarrubia-Martínez ${ }^{1}$, Ignacio Aguaded-Gómez², Águeda \\ Delgado-PonCE ${ }^{3}$ \\ ${ }^{1}$ Universidad de Huelva, (UHU), España. ORCID: https://orcid.org/0000-0002-2409- \\ 2224 \\ ${ }^{2}$ Programa Interuniversitario de Doctorado en Comunicación (UHU, US, UMA, UCA). \\ ORCID: https://orcid.org/0000-0002-0229-1118 \\ ${ }^{3}$ Universidad de Huelva y Universidad Internacional de Andalucía (UNIA). ORCID: htt- \\ ps://orcid.org/0000-0003-3261-2434 \\ Email: villarrubiaandrea@gmail.com; doctorado-comunicacion@uhu.es; agueda.del- \\ gado@dfesp.uhu.es
}

Abstract. Before the pandemic crisis, the irruption of the convergent scenario and the television digitalization forced the Latin American public television to develop strategies that consider new forms of audiovisual consumption and to make the resources profitable, such as the development of digital platforms and co-production with independent creators. Pakapaka, TVN and Señal Colombia coproduced children television programs with Chilean filmmakers that achieved a high audience and received acknowledgements at audiovisual festivals for their quality, their contribution to the local identity and Latin American own nature. Based on a content analysis carried out on tv shows aimed to children, present in both Chilean public digital platforms: CNTV Infantil (former Novasur) and TVN Kids, this study describes the collaborative model from the media literacy perspective, with emphasis on the diversity of children's programs, considering their origins, acquisition, financing, and characteristics of the protagonist's characters. The results indicate that the contents are varied and that the presence of female protagonists, native peoples and migrants, although incipient, constitutes a contribution to the identity of the continent's childhoods. In conclusion, it is essential that public service television can count on permanent funding that promotes the realization of relevant content for children, in accordance with their public service mission, especially in today pandemic crisis and confinement.

Keywords: Children's audiences, children's programs, convergence, digital platforms, public service television.

Resumen. Antes de la pandemia, el escenario convergente y la digitalización ya obligaron a las señales públicas latinoamericanas a desarrollar estrategias que considerarán 
las nuevas formas de consumo audiovisual y rentabilizarán los recursos, tales como el uso de plataformas digitales y la coproducción con independientes. Pakapaka, TVN y Señal Colombia colaboraron con realizadores chilenos en programas que lograron alta audiencia y recibieron premios en festivales audiovisuales por su calidad, aporte a la identidad local y sello latinoamericano. En el presente estudio, a partir de un análisis de contenido efectuado sobre programas compartidos por plataformas digitales públicas chilenas: TVN Kids y CNTV Infantil (antes llamada Novasur), se describe el modelo colaborativo desde la perspectiva educomunicativa, con énfasis en la diversidad de los programas infantiles, considerando su procedencia, adquisición, financiamiento y las características de sus protagonistas. Los resultados indican que los contenidos son variados y que la presencia de protagonistas femeninas, pueblos originarios y migrantes, aunque incipiente, constituye un aporte a la identidad de las infancias del continente. Como conclusión, es fundamental que las señales públicas puedan contar con financiamiento estable que potencie la realización de contenidos relevantes para la infancia, de acuerdo con su misión de servicio público, especialmente hoy en el contexto de la crisis sanitaria y el confinamiento.

Palabras clave: audiencias infantiles, convergencia, plataformas digitales, programas infantiles, televisión pública.

\section{JUSTIFICACIÓN TEÓRICA}

En los últimos años, poderosas "fuerzas globalizadoras", como denominan Potter y Steemers (2017) a los procesos tecnológicos asociados a la convergencia, así como la pandemia por la Covid-19, han cambiado la relación entre los medios y las audiencias. La convergencia entendida como proceso (Jenkins et al., 2013) es un fenómeno complejo que involucra a las audiencias, los géneros, los mercados y las industrias (Scolari, 2010). Esto significa que las audiencias pueden ver y también producir contenidos que estarán disponibles para todo el mundo a través de internet. Los contenidos pueden iniciarse en un medio como el cine para luego diversificarse en series de televisión, podcast y complementarse en otros dispositivos como videojuegos, aplicaciones, etc. Respecto de los mercados, ya no son locales, sino globales. Finalmente, las industrias son deslocalizadas, sin limitaciones de distribución y con una alta integración vertical.

La convergencia afecta no sólo la integración de contenidos y plataformas, sino que impacta en los hábitos de consumo de las audiencias, transformando a los públicos en generadores de contenidos (Aparici \& García-Marín, 2018). Como han advertido Hollis et al. (2020), las redes tienen luces y sombras: así como pueden ser utilizadas para mantener el contacto y los lazos en tiempos de aislamiento, también existen riesgos asociados a la sobreexposición y vulneración de derechos. Para participar plenamente de la sociedad digital, las audiencias requieren nuevas competencias conforme se consolidan narrativas transmedia (Pérez-Rodríguez et al., 2019).

Estos cambios que van más allá del ámbito tecnológico plantean desafíos para la misión de los medios de servicio público, la que es entendida por Unesco como la universalidad, el reflejo de la diversidad de los públicos -especialmente los desfavorecidos como los infantiles-, la independencia de los gobiernos y la diferenciación de otros medios comerciales (Banerjee y Seneviratne, 2006, p. 31). Los retos incluyen el tránsito hacia nuevas pantallas considerando innovar en los formatos y emitir en nuevos soportes para llegar a las audiencias. Según sugieren Azurmendi (2018) y Poell (2020), la convergencia obliga a repensar el quehacer de la industria, desde la medición de audiencias hasta las formas de narrar. Para Dhoest y te Walvaart (2017) y Potter y Steemers (2017) los medios de servicio público tienen el deber de profundizar su misión en el escenario convergente, considerando las oportunidades que ofrece internet para ampliar el acceso, diversificar los soportes de emisión e innovar en los formatos.

La televisión ha debido adaptarse a este escenario con sus propias dificultades como la falta de recursos económicos, la inestabilidad asociada a los cambios de gobierno y a la disminución en los índices de audiencia ante los grandes conglomerados de televisión infantil. Simultáneamente, la implementación de la televisión digital se desarrolla de manera desigual en los países del continente (CNTV y Gómez, 2018; Mastrini y Becerra,2019). En muchos de ellos se ha retrasado el encendido digital. Tampoco se ha cumplido con permitir el ingreso de nuevos actores como consignan Villarrubia-Martínez et al., 2019. Mientras en Brasil y Argentina, las emisoras públicas TV Brasil, Canal Encuentro y Pakapaka cuentan con una nueva vitrina de cara a las audiencias gracias a la TV digital.

Las nuevas formas de distribución de la televisión -gracias a internet- han cambiado las formas de consumo. Por ejemplo, el Video On Demand extiende la presencia de los contenidos en nuevas pantallas y amplía el visionado de los programas infantiles (Lotz, 2018; Corona y Orozco, 2020; Gallardo-Camacho et al., 2020). Estos autores, plantean los desafíos que imponen las nuevas formas de distribución más allá de los hábitos de los públicos, considerando aspectos como la medición 
tradicional de audiencia, la regulación, la tributación, entre otras. Michalis (2018) y Poell (2020) refieren el complejo proceso de ajuste de las señales públicas para coproducir con plataformas internacionales. Por ejemplo, los canales desarrollan estrategias que les permitan rentabilizar sus recursos siempre precarios, por medio de la generación de fondos alternativos - provenientes del cobro de licencias o concursos- y ampliar sus pantallas, a través del desarrollo de plataformas o con la exigencia de cuotas de programación infantil, (Steemers, 2019).

Parte de la misión de la televisión pública es generar contenidos que reflejen la identidad de las audiencias. Trappel (2019) y Steemers (2019) analizaron la dificultad de la televisión para incluir a las infancias en su diversidad, de allí la importancia de ampliar el rol de las audiencias infantiles. Si bien la convergencia permite en soportes digitales la participación de las audiencias, estudios como los de Medina y Ojer (2011), Gómez-Domínguez (2016) sobre las plataformas de $B B C$ y $T V 3$ de Cataluña; de Sánchez-Labella (2016) acerca de las plataformas dirigidas a niños en España y de Dhoest y te Walvaart (2017) sobre Ketnet la marca multiplataforma de Flandes, dan cuenta de que la participación del público se encuentra más en el ámbito de la interacción y de la co-creación que en la participación plena.

$\mathrm{Al}$ estilo de las señales públicas europeas, los canales latinoamericanos han desarrollado sus propias plataformas digitales o bien han aportado sus contenidos a plataformas estatales, que dan visibilidad a la producción audiovisual de cada país. En Chile, este proceso coincide con la discusión acerca de la misión de Televisión Nacional de Chile, TVN, como recoge Fuenzalida (2019). La señal estatal que hasta ahora se financiaba por vía publicitaria, ante la sostenida baja en los índices de audiencia, recibió una capitalización del Estado por 2500 millones de pesos, equivalentes a $\$ 3,9$ millones de dólares que estarían destinados a una nueva señal pública, cultural, educativa e infantil. En términos de programación, desde 2018 TVN ha vuelto a incorporar una franja de contenidos para niños, después de años de olvido de las audiencias infantiles, como se constata en los estudios realizados por el Consejo Nacional de Televisión [CNTV] en 2018 y 2019. Asimismo, desarrollaron la web TVN Kids, en sintonía con los tiempos actuales. Si bien se promociona como una plataforma, se trata de un espacio en la web del canal en la que se encuentra un repositorio de series, juegos y se dan a conocer los contenidos.

Durante la pandemia países como Japón, naciones europeas y del continente latinoamericano realizaron propuestas televisivas en apoyo al confinamiento entregando entretención y contención afectiva a los niños y sus familias y apoyando las estrategias educativas a dis- tancia (Yoshida, 2020; Carmona, 2020). TV Educa Chile surgió a partir de una alianza de los concesionarios de televisión -abierta nacional y regional-; del Consejo Nacional de Televisión y del Ministerio de Educación bajo la coordinación de TVN. Su objetivo es entregar programación infantil y en apoyo a la educación a través de las segundas señales de la TV digital de los distintos canales. Pese a que TV Educa Chile ha tenido buenos índices de audiencia y positiva valoración ciudadana según estudios de CNTV (2020), su continuidad, futura dependencia, gestión y financiamiento más allá de la crisis sanitaria se encuentran en evaluación.

Gran parte de la oferta de TVN Kids y de TV Educa Chile proviene del Consejo Nacional de Televisión. Este organismo autónomo estatal regula el funcionamiento de la televisión, administra el fondo de financiamiento para programas televisivos -el Fondo CNTV- y gestiona su propia oferta de contenidos infantiles, CNTV Infantil. Nacida en el año 2000 como Novasur, hoy transmite 14 horas de programación original mensual a través de 70 canales asociados -de TV abierta y por cable regional-. Asimismo, cuenta con materiales pedagógicos, emisión por streaming y con una videoteca virtual con más de 2000 programas para ver y descargar a través de la web https://cntvinfantil.cl/. Tanto los contenidos financiados por el Fondo CNTV como los realizados por CNTV Infantil constituyen una oferta de contenidos pública compartida por canales y plataformas.

Cabe preguntarse si estas estrategias -el desarrollo de plataformas digitales, la colaboración entre actores sectoriales del continente y el acceso a fondos concursables- permiten generar programas que sean una alternativa a la oferta internacional que proponen los grandes conglomerados y que sean contenidos que reflejen la diversidad e identidad de los niños chilenos y latinoamericanos, especialmente considerando la relevancia que ha adquirido la televisión en el contexto de la pandemia y el confinamiento.

\section{OBJETIVOS Y METODOLOGÍA}

A partir de las preguntas descritas, el estudio realizado tuvo como objetivo identificar y detectar en la diversidad de la programación de TVN Kids elementos del desarrollo colaborativo del sector audiovisual, tanto en el ámbito nacional -con los contenidos de CNTV Infantil y financiados por el Fondo CNTV- y del espectro latinoamericano.

El universo de programas estudiados corresponde a la oferta presente en el sitio TVN Kids entre 2018 y 2020, un total de 258 capítulos de 25 series, tanto nacionales 
Tabla 1. Instrumento de análisis cuantitativo.

\begin{tabular}{|c|c|c|c|}
\hline Variable & \multicolumn{3}{|c|}{ Categorías } \\
\hline Procedencia & - Nacional & - Extranjero & - Coproducción \\
\hline Financiamiento & - Fondo CNTV & $-\mathrm{Si} / \mathrm{No}$ & \\
\hline Empresa Productora & - Nombrar & & \\
\hline Tema & \multicolumn{3}{|c|}{-Nombrar (Noticiero; Ciencias; Aventuras; Cultura y Patrimonio; Formación ciudadana; Deportes; Artes) } \\
\hline Género audiovisual & - Ficción & -No Ficción & -Género mixto \\
\hline Técnica audiovisual & - Animación & - Acción real & -Técnica Mixta \\
\hline Público & $\begin{array}{l}\text { - Infantil (niños menores de } 6 \\
\text { años) }\end{array}$ & Niños (entre los 7 y 12 años) & Jóvenes (entre los 13 y los 17 años) \\
\hline Personajes protagónicos & $\begin{array}{l}\text { - Género protagonista } \\
\text { - Presencia pueblos originarios } \\
\text { - Presencia personajes con } \\
\text { discapacidad } \\
\text { - Presencia personajes migrantes }\end{array}$ & $\begin{array}{l}\text { Femenino/Masculino/Ambos } \\
\mathrm{Si} / \mathrm{No} \\
\mathrm{Si} / \mathrm{No} \\
\mathrm{Si} / \mathrm{No}\end{array}$ & \\
\hline
\end{tabular}

como internacionales. Para analizar los contenidos, se diseñó un instrumento que diera cuenta de la diversidad de los programas y de sus protagonistas. Sobre la totalidad de programas se registró el país de origen de los programas, si contaron con financiamiento del Fondo CNTV, la fecha en que se encontraban en la web y si estaban presentes en el repositorio de CNTV Infantil.

Para un análisis del contenido convergente se consideraron únicamente los programas compartidos por las plataformas TVN Kids y CNTV Infantil, correspondientes a 94 episodios de 8 series: 31 Minutos, Cehacheí, Niños inmigrantes, Tikitiklip, Los Tela, Horacio y Los Plasticines, Nanoaventuras y Puerto Papel. Sobre esta muestra se realizó un visionado de un capítulo de cada serie en la web de CNTV donde los contenidos permanecen de forma indefinida. A partir de la revisión de los capítulos se pudo determinar el tema, así como el género y la técnica audiovisual empleada en cada serie. Recogiendo el planteamiento de Fuenzalida (2019) sobre la diversidad de la representación infantil, se analizaron las características de sus protagonistas: si son niños o niñas; si pertenecen a pueblos originarios; si incluyen a migrantes o tienen alguna discapacidad. El instrumento utilizado está descrito en la Tabla 1.

Con el propósito de ver el tránsito de los contenidos en la plataforma de TVN Kids, el análisis se aplicó en tres períodos: marzo de 2018, marzo de 2019 y marzo de 2020, considerando los programas presentes en su web, como se describe la Tabla 2.

\section{RESULTADOS}

Como se señaló anteriormente, la oferta de contenidos de la plataforma TVN Kids entre 2018 y 2020 corres- ponde a 258 episodios de 25 series, tanto nacionales, como extranjeras y coproducciones internacionales. Los programas del país son mayoritarios: 17 de las 25 series son producidas en Chile, lo que corresponde al $68 \%$ del total de programas. Respecto de los capítulos, 177 son nacionales. Los contenidos extranjeros equivalen a 5 series con 49 capítulos, como puede verse en la Tabla 2. Entre ellas, destacan la premiada serie Los cuentos de la casa del árbol, de Pakapaka, Mily Preguntas de Francia y Mónica Toys de Brasil, Raro pero cierto, de Estados Unidos y El Chavo del 8 de México.

La mayoría de los espacios de TVN Kids no son realizaciones propias del canal, sino contenidos financiados por el Fondo del Consejo Nacional de Televisión: 16 series, correspondientes al 64\% de los programas y 151 capítulos, como ejemplifica la Tabla 4 . Son realizados por productoras independientes, ya han sido emitidos anteriormente por otros canales de la TV generalista, si bien no en una franja u horario específicamente infantil. Igualmente, algunos de los contenidos están presentes en la web de CNTV Infantil, en Plataforma Audiovisual y, más recientemente, se están emitiendo a través de $T V$ Educa Chile.

Entre las productoras existe diversidad, ya que 13 empresas entregan contenidos al canal: Zumbástico Studios está detrás de 3 programas: Achú, Zumbástico Fantástico y Horacio y Los Plasticines. TVN está involucrada directamente en la producción de tres programas: Kunga, actitud animal, Tikitiklip, realizado en conjunto con Ojitos producciones y La aventura de crecer, con el organismo estatal de educación parvularia JUNJI. El resto de las empresas como Aplaplac, Invercine, Punkrobot, Cábala, Vivienne Barry, entre otras, produjeron una serie cada una.

Así como se ha producido la colaboración entre señales públicas del continente, también los canales 
Tabla 2. Programas Plataforma TVN Kids 2018-2020.

\begin{tabular}{|c|c|c|c|c|c|}
\hline Nombre de la serie & País & Fondo & Emisión & CNTV Infantil & Capítulos \\
\hline Kunga, actitud animal & Chile & No & 2018 & No & 2 \\
\hline Ceacheí & Chile & $\mathrm{Si}, 2014$ & 2018 & Sí & 13 \\
\hline Niños inmigrantes & Chile & $\mathrm{Si}, 2012$ & 2018 & Sí & 13 \\
\hline Horacio y Los Plasticines & Chile & $\mathrm{Si}, 2013$ & 2018 & Sí & 14 \\
\hline Los Tela & Chile & $\mathrm{Si}, 2013$ & 2018 & Sí & 12 \\
\hline Los fantásticos viajes de Ruka & Chile & Si, 2015 & 2018-2019 & No & 12 \\
\hline Los próceres más posers & Chile & $\mathrm{Si}, 2014$ & 2018-2019 & No & 10 \\
\hline Achú & Chile & No & 2018-2019 & No & 14 \\
\hline Nanoaventuras & Chile & $\mathrm{Si}, 2011$ & $2018-2019$ & Sí & 20 \\
\hline Puerto Papel & $\begin{array}{c}\text { Coproducción Chile, Brasil, Colombia y } \\
\text { Argentina }\end{array}$ & $\mathrm{Si}, 2012$ & $2018-2019$ & Sí & 20 \\
\hline Mónica Toys & Brasil. & No & 2019 & No & 16 \\
\hline Nuku, Nuku & Chile & $\mathrm{Si}, 2014$ & 2019 & No & 4 \\
\hline Los cuentos de la casa del árbol & Argentina & No & 2019 & No & 6 \\
\hline Raro pero cierto & Estados Unidos & No & 2019 & No & 13 \\
\hline Bebé en camino & Coproducción Chile, Corea, Camboya e Italia. & No & 2019 & No & 2 \\
\hline Tikitiklip & Chile & $\mathrm{Si}, 2007$ & 2019-2020 & Sí & 4 \\
\hline Mily Preguntas & Francia & No & $2019-2020$ & No & 4 \\
\hline Cantando aprendo a hablar & Chile & $\mathrm{Si}, 2007$ & $2019-2020$ & No & 14 \\
\hline Yo Pippo & Chile & Si, 2016 & 2020 & No & 10 \\
\hline Guitarra y Tambor & Chile & $\mathrm{Si}, 2016$ & 2020 & No & 2 \\
\hline Locoslab & Coproducción internacional & $\mathrm{Si}, 2017$ & 2020 & No & 10 \\
\hline La aventura de crecer & Chile & No & 2020 & No & 10 \\
\hline El Chavo del 8 & México & No & 2020 & No & 10 \\
\hline 31 Minutos & Chile & $\mathrm{Si}, 2012$ & $2018-2020$ & Sí & 20 \\
\hline Zumbástico fantástico & Chile & Si, 2007 & $2018-2020$ & No & 3 \\
\hline Total & 258 & & & & \\
\hline
\end{tabular}

colaboran con productoras locales, tal como documenta Gutiérrez-González (2019). Las coproducciones internacionales son iniciativas que permiten potenciar los recursos financieros y profesionales de los canales y las productoras de distintos lugares del mundo. Se trata de realizaciones que involucran a empresas independientes que postulan a fondos concursables y se emiten por los canales públicos de diferentes países. A través de TVN Kids se han emitido 3 coproducciones internacionales con un total de 32 capítulos. Dos de ellas han sido financiadas por la colaboración entre estos actores:
Puerto Papel, que fue realizada por Zumbástico Studios con la participación de Gloob de Brasil, Señal Colombia y Pakapaka. Locos Lab, es una realización de Señal Colombia y la productora Dinogorila Creative Lab. La tercera serie, Bebé en Camino es una realización de Mi Chica Producciones que ganó el premio EMMY por los programas Con qué sueñas y Sueños Latinoamericanos, ambos financiadas por el Fondo CNTV. Bebé en camino relata la llegada a la familia de un nuevo integrante desde el punto de vista de su hermana, es una coproducción entre Mi Chica y TVN con EBS Corea, TVK Camboya,
Tabla 3. Procedencia series TVN Kids, en número.

\begin{tabular}{lcc}
\hline Procedencia & Series & Capítulos \\
\hline Programas nacionales & 17 & 177 \\
Internacionales & 5 & 49 \\
Coproducción Internacional & 3 & 32 \\
Total & 25 & 258 \\
\hline
\end{tabular}

Tabla 4. Programas financiados Fondo CNTV, en número.

\begin{tabular}{lcc}
\hline Procedencia & Series & Capítulos \\
\hline Programas nacionales & 16 & 151 \\
Internacionales & 0 & 0 \\
Coproducción Internacional & 2 & 30 \\
Total & $\mathbf{1 8}$ & $\mathbf{1 8 1}$
\end{tabular}


Tabla 5. Premios Programa Puerto Papel 2016-2019.

\begin{tabular}{|c|c|c|c|}
\hline Festival & País & Año & Premio \\
\hline Chilemonos & Chile & 2016 & Serie animada \\
\hline TAL & & 2016 & $\begin{array}{c}\text { Mejor Producción } \\
\text { infantil }\end{array}$ \\
\hline Prix Jeunesse Internacional & Alemania & 2016 & Beyond TV \\
\hline Festival ANIMA & Argentina & 2017 & \\
\hline Prix Jeunesse Iberoamericano & Brasil & 2017 & $\begin{array}{c}2^{\circ} \text { lugar Ficción } 7 \text { a } \\
11 \text { años }\end{array}$ \\
\hline Prix Jeunesse Iberoamericano & Brasil & 2019 & $\begin{array}{c}2^{\circ} \text { lugar Ficción } 7 \text { a } \\
11 \text { años }\end{array}$ \\
\hline
\end{tabular}

Thai PBS, ShowLab de Italia y fue premiado en Prix Jeunesse Iberoamericano 2017.

Respecto del Fondo CNTV, en su primera temporada, Puerto Papel recibió de parte del Estado algo más de 131 millones de pesos y en la segunda temporada, 105 millones (CNTV 2017, p. 94). Actualmente la serie se transmite a través de Pakapaka, Señal Colombia y otros 50 países. Por su calidad el programa ha recibido reconocimientos en diversos festivales nacionales e internacionales, incluyendo el Beyond TV en el Prix Jeunesse Internacional, tal como se detalla en la Tabla 5. Por otra parte, Locos Lab, se adjudicó el Fondo CNTV en 2017 por un monto de 219 millones (CNTV 2017, p. 102).

El análisis de contenido realizado sobre los 94 capítulos de 8 series programadas por TVN Kids y presentes en la web de CNTV Infantil, buscó conocer la diversidad de los programas. Respecto del formato: género y técnica audiovisual, 5 de las series con 60 capítulos corresponden a ficciones, todas animaciones o de técnica mixta que combina títeres y animación. Tikitiklip y Horacio y Los Plasticines son animaciones con técnicas mixtas, dirigidas a niños de entre 3 y 6 años. Para el público de entre 7 y 12 años hay programas de ficción como las animaciones Puerto Papel, Nanoaventuras y 31 Minutos.
Además, hay programas de no ficción documental de acción real como Ceacheí y Los Tela. Niños Inmigrantes estaría dirigido a un público más adolescente, entre 13 y 17 años.

Como se observa en la Tabla 6 los temas de los programas son variados. Mientras Horacio y Los Plasticines busca desarrollar habilidades ciudadanas en los más pequeños, Tikitiklip se concentra en el patrimonio material de los pueblos originarios, a través de las aventuras de una niña en el Museo Precolombino. 31 Minutos es un noticiero dirigido a niños y niñas conducidos por títeres que abordan desde el humor diversas situaciones. Puerto Papel es una serie en la que Matilde, una inquieta niña descubre cada día un nuevo y extraño superpoder que la lleva a experimentar divertidas aventuras acompañada de sus inseparables amigos y su abuelo. Nanoaventuras es un programa de ciencias en el que un niño, su hermana adolescente y un despistado científico logran hacerse pequeños para averiguar distintos temas relacionados con el cuerpo humano. Ceacheí presenta a niños de distintas disciplinas deportivas en su cotidianidad. Niños Inmigrantes da a conocer la realidad de niños que han llegado al país, los que cuentan sus experiencias y el proceso de adaptarse a una nueva cultura sin perder sus tradiciones. Es especialmente interesante la interacción que tienen los niños con los artistas plásticos en Los Tela, programa en el que intercambian experiencias y trabajan como pares en el desarrollo de una obra artística.

El último aspecto estudiado fue la diversidad de los protagonistas de las series, según dimensiones como su género y pertenencia a pueblos originarios o condición de migrantes. Respecto del género, la mayoría de los programas tiene protagonistas masculinos y femeninos (63 capítulos de 5 series). Ello se asocia a que los programas tienen más de un personaje protagónico y esos grupos suelen ser mixtos, como es el caso de 31 Minutos, Nanoaventuras o Puerto Papel. Existen también capítulos de series con protagonistas masculinos: 14 episodios

Tabla 6. Resumen diversidad de programas TVN Kids Plataforma CNTV Infantil.

Base 94 capítulos

\begin{tabular}{lcccc}
\hline Programa & Género & Técnica & Público Objetivo & Tema \\
\hline 31 Minutos & Ficción & Mixta & 7 a 12 años & Noticiero \\
Nanoaventuras & Ficción & Animación & 7 a 12 años & Ciencias \\
Puerto Papel & Ficción & Animación & 7 a 12 años & Aventuras \\
Tikitiklip & Ficción & Animación & 3 a 6 años & Cultura y Patrimonio \\
Horacio y Los Plasticines & Ficción & Mixta & 3 a 6 años & Formación ciudadana \\
Ceacheí & No Ficción & Acción real & 7 a 12 años & Deportes \\
Los Tela & No Ficción & Acción real & 7 a 12 años & Artes \\
Niños Inmigrantes & No Ficción & Acción real & 13 a 17 años & Cultura y Patrimonio \\
\hline
\end{tabular}


Tabla 7. Protagonistas Programas, según géneros.

\begin{tabular}{ccc}
\hline \multicolumn{3}{c}{ Base 94 capítulos } \\
\hline Masculinos & Femeninos & Mixto \\
\hline 14 & 17 & 63 \\
\hline
\end{tabular}

de las series documentales Cehacheí, Niños Inmigrantes y Los Tela. Mientras que 17 de esas series son episodios protagonizados por mujeres. Del mismo modo, la protagonista de Tikitiklip es una niña.

Respecto de la presencia de migrantes y pueblos originarios, se trata de dimensiones incipientes, ya que mínimamente se recogen en las series, salvo en algún capítulo documental de Cehacheí. Sin embargo, cabe destacar que programas como Niños Inmigrantes está exclusivamente centrado en este grupo, así como Tikitiklip en dar a conocer el patrimonio de los pueblos originarios, tanto de Chile como del continente, lo que sin duda es un aporte a que los niños puedan reconocerse en pantalla.

\section{DISCUSIÓN Y CONCLUSIONES}

En tiempos de digitalización y convergencia la misión de los medios de servicio público que incluye lograr la universalidad, acoger la diversidad de sus públicos e innovar en los formatos es posible, si se sortean los desafíos políticos, tecnológicos, presupuestarios, regulatorios, de medición de audiencias y narrativos (Dhoest y te Walvaart, 2017; Potter y Steemers, 2017; Poell, 2020).

Los canales de televisión toman estrategias para adaptarse a este escenario convergente, tales como el desarrollo de sus propias plataformas digitales y la colaboración con productoras locales y con otras señales públicas del continente. Respecto de TVN, Fuenzalida (2019) propone una serie de medidas para preservar su misión como medio público, entre ellas servir de reflejo a la diversidad de sus audiencias infantiles, aprovechando las pantallas anexas.

El uso de plataformas permite entender los nuevos consumos deslocalizados y en diferido de las audiencias (Gallardo-Camacho et al., 2020; Corona y Orozco, 2020). Sin embargo, la sola existencia de plataformas no garantiza hacer un uso interactivo de ellas (Medina y Ojer, 2011; Sánchez-Labella, 2016; Gómez-Domínguez, 2016). Tampoco la presencia de los contenidos en línea aumenta por sí misma la participación de las audiencias (Dhoest y te Walvaart, 2017).

Considerando que más de 258 episodios de 25 programas han formado parte de TVN Kids es lamentable- mente que como plataforma digital no cumpla con servir de repositorio para todos los contenidos programados, ni cuente con espacios dedicados a la interacción, ni menos a la participación de sus audiencias.

En este estudio se muestra cómo la oferta de TVN en diferentes soportes se ha ido consolidando como espacio dedicado a los niños y a servir de vitrina a contenidos financiados con recursos públicos, después de años de ausencia de contenidos infantiles nacionales en la televisión y en otras pantallas. Resulta relevante que la misión de entregar contenidos infantiles de calidad se realice en colaboración con otras señales y plataformas públicas chilenas como TV Educa Chile y CNTV infantil. Esta cooperación se basa en el financiamiento del Fondo CNTV que es central para proveer contenidos a todas las señales y plataformas públicas y para la TV abierta nacional, local y regional. CNTV Infantil aporta contenidos y sirve como repositorio permanente de contenidos y de otros materiales pedagógicos.

Respecto de los nuevos contenidos incorporados en 2020 por TVN Kids, todavía se trata en su mayoría de espacios financiados en los concursos del Fondo del CNTV, siendo menor el impulso productor del canal. La producción propia, que visibiliza a las infancias sus propias identidades es el corazón de las señales de servicio público, como plantean Dhoest y te Walvaart (2017). Si bien es deseable que el canal produzca por sí solo, es cierto que forma parte de una cadena de colaboración con productoras locales (Gutiérrez-González, 2012). El canal sirve como una vitrina más para los contenidos realizados en forma independiente en el país y permite atender la demanda de las audiencias infantiles hacia la televisión abierta, al diversificar sus contenidos.

$\mathrm{Al}$ analizar los formatos audiovisuales (género y técnicas) de los contenidos es visible que no se utiliza la narrativa transmedia, en la línea que propone Azurmendi (2018) y no se promueven las competencias mediáticas que sugieren Pérez-Rodríguez et al., 2020, por lo que sería deseable contar con propuestas de este tipo en el futuro.

En términos de diversidad, siguiendo a Fuenzalida (2019) igualmente, de forma incipiente da cabida a infancias que no están habitualmente presentes en pantalla: niñas, inmigrantes y pueblos originarios. Esta presencia viene a corregir la desigualdad que enfrentan niños y niñas invisibilizados en la pantalla como mencionan Steemers (2019) y Trappel (2019).

Aun cuando la coproducción internacional no es todavía un fenómeno masivo que permita dotar a las pantallas de contenidos con carácter latinoamericano, ha permitido el desarrollo de audiovisuales de primer nivel, reconocidos tanto por el público infantil en los 
diferentes países en los que se emiten los programas, como en destacados festivales internacionales. Programas producidos en colaboración por productoras locales y señales públicas de varios países, como Puerto Papel, son iniciativas para promover considerando las dificultades que supone para los canales públicos producir para grandes distribuidores como Netflix, (Lotz, 2018; Michalis, 2018).

Los cambios políticos a gobiernos con diferente signo político en varios países han afectado la gestión y financiamiento público de los canales: mientras en Argentina se restringió el personal y el presupuesto a las señales públicas, en Brasil se puso fin al convenio del Estado con TV Escola y en Colombia se discuten leyes para cambiar el financiamiento proveniente de las licencias de televisión. Hoy los canales públicos precisan de nuevos recursos, económicos y humanos para desarrollar ofertas adecuadas en estos nuevos entornos, tanto en el ámbito de la producción para público infantil, como en la generación de contenidos acordes con la convergencia: formatos crossmedia y otras narrativas que sean innovadoras y atractivas para los usuarios. Esto es especialmente deseable para permitir la fidelización de los más pequeños con la señal pública, en tanto niños y niñas están acostumbrados a acceder a contenidos desde diferentes dispositivos, en cualquier momento y lugar. El contexto de la pandemia obligará a revisar del rol de la televisión y sus pantallas anexas en apoyo a la educación y a la contención socioemocional de las infancias.

\section{REFERENCIAS}

Aparici, R., \& García-Marín, D. (2018). Prosumidores y emirecs: Análisis de dos teorías enfrentadas [Prosumers and emirecs: Analysis of two confronted theories]. Comunicar, (26)55, 71-79. http://www.doi. org/10.3916/C55-2018-07

Azurmendi, A. (2018). Reconectar con la audiencia joven. Narrativa transmedia para la transformación de la televisión de servicio público en España, Francia, Alemania y Reino Unido. Revista Latina de Comunicación Social, 73, 927-944. https:/doi. org/10.4185/RLCS-2018-1289

Banerjee, I., \& Seneviratne, K. (Eds.), Radiotelevisión De Servicio Público: Un Manual De Mejores Prácticas, 42-72. UNESCO. http://bit.ly/35DEFST

Carmona, B. (2020). COVID-19 and children's media in Latin and South America. Responses on TV screens, platforms, and video channels. TelevIZIon, 33/2020/E, 34-36. https://bit.ly/2RvekSi
Consejo Nacional de Televisión (Ed.) (2018). Resumen histórico Encuesta Nacional de Televisión. Consejo Nacional de Televisión. http://bit.ly/2tboazM

Consejo Nacional de Televisión (Ed.) (2017). Fichas de programas Fondo CNTV. Consejo Nacional de Televisión. https://bit.ly/3duAVbf

Consejo Nacional de Televisión (Ed.) (2019). Anuario Estadístico Oferta y Consumo de Televisión 2018. Consejo Nacional de Televisión. http://bit.ly/2PAsLDo

Consejo Nacional de Televisión, \& Gómez, G. (2018). Transición a la TV digital y diversidad en Latinoamérica. Consejo Nacional de Televisión. http://bit. ly/35B51on

Consejo Nacional de Televisión (Ed.) (2020). Evaluación de iniciativa TV Educa Chile. https://bit.ly/3pyD6jq

Corona, J.M., \& Orozco, G. (2020). El desafío de la comprensión de las audiencias contemporáneas el Video on Demand y sus posibilidades para analizar a sus audiencias. En G. Orozco (Coord.), Televisión en tiempo de Netflix. Una nueva oferta mediática, (pp. 213-243) Universidad de Guadalajara.

Dhoest, A., \& te Walvaart, M. (2018). Convergence and Participation in Children's Television. The case of Flemish Public Television. En G. Ferrel-Lowe, H. van den Bulck, \& K. Donders (Eds.), Public Service Media in the Networked Society (pp. 245-257). Nordicom. https://bit.ly/2ZT8Y94

Fuenzalida, V. (2019). Análisis y propuestas para actualizar la misión pública de TVN. Perspectivas de la comunicación, 12(1), 315-339. https://bit.ly/2UplicD

Gallardo-Camacho, J., Lavín, E., \& Sierra-Sánchez, J. (2020). Los programas infantiles de televisión y su consumo en diferido en España. Icono 14, 18(1), 155178. https://doi.org/10.7195/ri14.v18i1.1387

Gómez-Domínguez, P. (2016). Era digital y televisión autonómica: un estudio comparativo de las plataformas web, aplicaciones móviles y redes sociales de TV3 y BBC One. Communication \& Society. 29(4), 85-106. https://doi.org/10.15581/003.29.4.sp.85-106

Gutiérrez-González, C. (2019). La relación entre los creativos independientes de la televisión pública y la audiencia infantil. Anagramas Rumbos y Sentidos de la Comunicación. 18(35), 39-55. http://doi. org/10.22395/angr.v18n35a3

Jenkins, H., Ford, S., \& Green, J. (2013). Cultura transmedia. La creación de contenido y valor en una cultura en red. Gedisa.

Lotz, A. (2018). Portals a treatise on Internet DistributedTelevision. Michigan Publishing, University of Michigan Library. https://doi.org/10.3998/mpub.9699689

Mastrini, G., \& Becerra, M. (2019). La convergencia de medios, telecomunicaciones e internet en la perspec- 
tiva de la competencia: hacia un enfoque multicomprensivo. Cuadernos de Discusión de Comunicación e Información. UNESCO. http://bit.ly/36AnLG1

Medina, M., \& Ojer, T. (2011). La transformación de las televisiones públicas en servicios digitales en la $\mathrm{BBC}$ y RTVE [The Transformation of Public TV Companies into Digital Services at the BBC and RTVE]. Comunicar, 36, 87-94. https://doi.org/10.3916/C36-2011-02-09

Michalis, M. (2018). Distributions Dilemmas for Public Service Media. Evidence from the BBC. En G. FerrelLowe, H. van den Bulck, \& K. Donders (Eds.), Public Service Media in the Networked Society (pp. 195-210). Nordicom. https://bit.ly/32DA1W.

Pérez-Rodríguez, M. A., Pérez-Escoda, A., \& SánchezLópez, I. (2019). De lo audiovisual a lo transmedia. Competencias para las nuevas narrativas de los prosumidores en el ecosistema digital. En L.M., RomeroRodríguez \& D. Rivera-Rogel, La comunicación en el escenario digital: Actualidad, retos y prospectivas (pp. 119-146). Pearson Education. https://bit.ly/2XicWWS

Poell, T. (2020). Three Challenges for Media Studies in the Age of Platforms. Television \& New Media, 21(6), 650-657. https://doi.org/10.1177/I1527476420918833

Potter, A., \& Steemers, J. (2017). Children's television in transition: policies, platforms, and production. Media International Australia, 163(1), 6-12. https://doi. org/10,1177/1329878X17693936

Sánchez-Labella Martín, I. (2016) La calidad de los dibujos animados en internet. Clan RTVE, Neox Kidz y Boing: Plataformas de entretenimiento para el público infantil. Index Comunicación, 6(2), 173-190. http://bit. ly/37RRKJD

Scolari, C. (2010). Convergencia, medios y educación. Convergencia de medios: nuevos desafíos para la educación en Latinoamérica. Serie Seminario. RELPE. https://bit.ly/3bYw836

Steemers, J. (2019). Invisible children. Inequalities in the provision of screen content for children. En J. Trappel (Ed.), Digital Media Inequalities. Policies against divides, distrust, and discrimination (pp. 179-192). Nordicom. https://bit.ly/36QIiXN

Trappel, J. (2019). Inequality, (new) media and communications. En J. Trappel (Ed.), Digital Media Inequalities. Policies against divides, distrust and discrimination (pp. 9-30). Nordicom. bit.ly/3c4tPdo

Villarrubia-Martínez, A., Aguaded-Gómez, I., \& Delgado-Ponce, A. (2019). Implementación de la Televisión digital en Chile: ¿una oportunidad real para la TV Comunitaria? Chasqui, 140, 279-296. https://doi. org/10.16921/chasqui.v0i140.3878

Yoshida, Y. (2020). NHK makes decisive responses to the coronavirus pandemic. TelevIZIon, 33/2020/E, 45. https://bit.ly/33AlwlN 